\title{
Todavía sobre la objetividad periodística: hacia la superación de un paradigma fracturado*
}

\author{
Juan Ramón MuÑOZ TORRES \\ Universidad Complutense de Madrid \\ jmtorres@pdi.ucm.es
}

Recibido: 13 de marzo de 2012

Aceptado: 15 de octubre de 2012

\begin{abstract}
Resumen
Desde finales del siglo XIX, la teoría de la objetividad fue generando el que llegaría a ser el paradigma dominante en el ejercicio periodístico, así como en la reflexión académica sobre él. Sin embargo, durante las últimas décadas del siglo $\mathrm{XX}$, fue surgiendo un creciente número de académicos y periodistas que rebatieron las tesis fundamentales de este paradigma, hasta llegar a ponerlo en crisis. Desde entonces, ningún otro concepto ha suscitado tanta controversia como el concepto de objetividad, que todavía origina debates, no sólo interminables, sino inconcluyentes. En este artículo se aborda la cuestión desde una perspectiva habitualmente ignorada: la de los fundamentos filosóficos de la teoría objetivista y su relación con el positivismo, cuyas raíces se remontan al empirismo inglés. Analizando tales fundamentos, se argumenta que la teoría objetivista parte de una pregunta mal concebida, lo cual explica por qué estamos ante un paradigma fracturado, que, pese a ello, sigue todavía firmemente arraigado en nuestras mentes. Finalmente, se apuntan algunas vías hacia su posible superación.

Palabras clave: verdad, objetividad, positivismo, relativismo, realismo, distinción hecho-valor, dicotomía información-opinión.
\end{abstract}

\section{Still with journalistic objectivity: toward overcoming a paradigm fractured}

\begin{abstract}
Since the end of the nineteenth century, the theory of objectivity has been creating what it would be the prevailing paradigm both in journalistic practice and in the scholarly reflection upon it. However, during the last decades of the twentieth century, an increasing number of communication scholars and practitioners began to contest the main notions embedded in this paradigm, which was put in crisis. Since then, no other concept has stimulated as much controversy as the concept of objectivity, which still gives origin to debates not only endless, but also inconclusive. This article addresses the topic from an often neglected perspective, namely, that of the philosophical underpinnings of the theory of objectivity and its bearing upon the positivist presumptions, dated back to the empiricist tradition. By putting those foundations into scrutiny, it is argued that the theory of objectivity stems from an ill-conceived question. This accounts why this is a fractured paradigm, which, nonetheless, still remains firmly entrenched in our minds. Lastly, some hints are suggested as to how to overcome and replace it.
\end{abstract}

Keywords: truth, objectivity, positivism, relativism, realism, fact-value distinction, news-opinion dichotomy.

Referencia normalizada: MUÑOZ TORRES, Juan Ramón (2012): “Todavía sobre la objetividad periodística: hacia la superación de un paradigma fracturado". Estudios sobre el mensaje periodístico, vol. 18, núm. 2 (julio-diciembre), págs.: 833-854. Madrid, Servicio de Publicaciones de la Universidad Complutense.

* Una versión anterior de este texto fue presentada en la sección Philosophy of Communication del Congreso Internacional organizado por la European Communication Research and Education Association (ECREA), que se celebró en Octubre de 2010 en Hamburgo. Quisiera manifestar mi agradecimiento a los participantes en la sesión de presentación de la ponencia por sus valiosas preguntas y comentarios y, muy particularmente, al presidente de la sección, Dr. Johan Siebers, por su interés y apoyo. 
Sumario: 1. Introducción. 2. Un concepto insuficientemente definido. 3. Nociones recurrentes en las principales definiciones de objetividad. 4. Premisas filosóficas subyacentes en la teoría de la objetividad; 4.1. El positivismo como origen de la teoría de la objetividad y su vigor contemporáneo; 4.2. La dicotomía hecho-valor: una distinción tan clara como falsa; 4.2.1. Por qué es imposible una percepción pura de hechos desprovista de conceptos previos; 4.2.2. Por qué es imposible seleccionar hechos sin valores. 4.2.3. Las contradicciones inherentes a la teoría de la objetividad. 5. Las contradicciones inherentes a la teoría de la objetividad; 5.1. El intento de sustituir verdad por objetividad; 5.2. Un movimiento pendular desde la objetividad: el relativismo; 5.3. Objectivismo y relativismo, ¿dos caras de la misma moneda?; 5.4. ¿Puede haber una vía media entre positivismo y relativismo?. 6. Conclusiones. 7. Referencias bibliográficas.

\section{Introducción}

Desde finales del siglo XIX, la teoría de la objetividad ha sido considerada la piedra angular del periodismo. Es interminable la nómina de autores que, de una manera u otra, así lo han subrayado, en particular, dentro del ámbito anglosajón. Como sostiene SCHUDSON (1978: 9), la objetividad ha llegado a ser "el emblema" del periodismo norteamericano. En palabras de RoSEN (1993: 48), es "quizá la contribución más importante que el periodismo americano ha hecho al resto del mundo. Cualquiera que trate de pensar sobre nuestro modo de hacer periodismo debe enfrentarse con este concepto, que es esencial para entender el modo en que la prensa americana se ve a sí misma y el modo en que América ve a la prensa". MinDICH (1998), por su parte, va un paso más allá cuando afirma que, sin el concepto de objetividad, el periodismo norteamericano no puede ser entendido en absoluto. Y esta afirmación puede ser extendida, en parte, a muchos otros países influidos por él ${ }^{1}$.

Ahora bien, este consenso sobre el papel predominante que ha jugado históricamente el concepto de objetividad puede inducir a pensar, de manera errónea, que esta teoría ha sido sostenida casi unánimemente, tanto por académicos como por periodistas, y que ha mantenido su poderosa influencia hasta el presente. Sin embargo, no es éste el caso. Como Durham señala (1998: 117) con acierto "durante la mayor parte de este siglo [se refiere al XX], la objetividad ha sido considerada un marchamo de excelencia informativa [...]. Sin embargo, a pesar de la longevidad de esta tradición, recientemente toda la epistemología de la 'objetividad' en periodismo ha caído en desgracia. Los análisis contemporáneos de los medios informativos han convertido la que una vez fue transparente noción de objetividad en un asunto de investigación fuertemente controvertido. Examinados rigurosamente, sus fundamentos filosóficos han sido desafiados, reevaluados y, finalmente, rechazados".

Otros muchos autores comparten esta visión. Rosen (1993: 48), por ejemplo, escribe: “[...] la objetividad se está quebrando de un montón de maneras diferentes. Es un mecanismo que ya no funciona como solía hacerlo. Hay una buena dosis de preo-

\footnotetext{
${ }^{1}$ A pesar de la gran influencia del periodismo norteamericano en la práctica profesional de la mayor parte de los países occidentales, la evolución y el peso de la teoría de la objetividad están lejos de ser homogéneos en todos ellos. De hecho, algunos autores han advertido que, mientras los periodistas norteamericanos todavía mantienen normas de objetividad, equilibrio y neutralidad, sus colegas europeos no las valoran tanto y tienden a pensar que es más importante que ellos defiendan determinadas ideas y valores (DONSBACH, 1995).
} 
cupación y de confusión sobre este término entre los propios periodistas. Casi siempre que alguien en periodismo usa la palabra objetividad ésta va seguida de la coletilla: 'cualquiera que sea lo que esta palabra significa', lo que indica la existencia de un problema conceptual que se transparenta". Por su parte, DONSBACH y KLETT (1993: 53) observan atinadamente que "probablemente no hay ningún otro término [quizá la palabra adecuada sea 'concepto'] que haya suscitado tanta deliberación y controversia dentro y fuera de la profesión periodística como el término 'objetividad" (la acotación es mía).

Efectivamente, el paradigma de la objetividad ha venido sufriendo un progresivo declive durante las últimas décadas, hasta el punto de que puede decirse, con verdad, que se trata de un paradigma fracturado (por usar la conocida expresión de Entman). Sin embargo, no es menos cierto que aún no ha surgido un nuevo paradigma que lo sustituya. Por eso, la objetividad todavía sirve en parte -como recuerda GLASSER (1992: 176)- como "un marco de referencia que los periodistas usan para orientarse a sí mismos en la redacción y en la sociedad", porque lleva dentro de él "una visión particular del periodismo y de la prensa".

La situación actual de "interregno" entre un paradigma que no acaba de ser superado por otro nuevo, pero que sigue en lenta decadencia se aprecia -entre otros fenómenos- en que el tema de la objetividad sigue generando amplia bibliografía y dando lugar a controversia. Este carácter polémico se manifiesta, con frecuencia, en forma de debates interminables y -lo que es peor- inconcluyentes y aporéticos. Los que están a favor se enzarzan en disputas con sus detractores, y unos y otros se arrojan los mismos argumentos y refutaciones una vez tras otra, dejando la discusión, a la postre, en el mismo punto de partida en el que empezó. Este esfuerzo, tan extenuante como infructuoso, recuerda en cierta manera los sufrimientos de Sísifo, castigado a subir una pesada roca a una colina, que volvía a caer al llegar a la cima, y a repetir esa tarea durante toda la eternidad.

Así las cosas, a estas alturas, es difícil y arriesgado intentar ofrecer una contribución enriquecedora sobre esta cuestión, sin correr el peligro de incurrir, una vez más, en una enésima variación sobre el mismo tema. Por eso, el propósito de este artículo no es hacer una valoración general del status quaestionis, acerca de lo cual existen muy buenos trabajos (e.g., Schudson, 1978; Durham, 1998; RYAN, 2001; WARD, 2005). Lo que me propongo aquí, en cambio, es aventurar la tesis de que la causa última que explica por qué este debate todavía sigue siendo interminable e inconcluyente es porque está conceptualmente mal planteado desde su mismo origen.

Precisando un poco más: si se va al núcleo del problema, hay una pregunta clave que subyace a toda la controversia sobre la objetividad. De manera simplificada, aunque no simplista -espero-, esta pregunta podría ser formulada en los siguientes términos: cómo podemos nosotros, siendo sujetos, llegar a conocer objetivamente. Permítaseme decir -quizá con algo de temeridad- que tal pregunta es absurda e induce a confusión. Para mostrarlo, en las páginas que siguen, voy a tratar de argumentar que:

a) La objetividad no solo no es un ideal imposible -como se ha afirmado incansablemente-, sino que se trata más bien de una cuestión mal concebida, que arrastra los presupuestos erróneos de su origen: el positivismo. 
b) El concepto de objetividad ha logrado reemplazar, con el tiempo, a otro más fundamental, el de verdad, lo cual ha hecho que se convierta en confuso y falaz.

c) Se necesita con urgencia un nuevo paradigma, basado en una rehabilitación del concepto realista de verdad, que sustituya al anterior, ya agotado, con el fin de remediar sus deficiencias congénitas.

Probablemente sea superfluo añadir que mi propósito no es, en modo alguno, ofrecer una solución para un asunto tan complejo como el que nos ocupa. Sería pretencioso por mi parte aspirar a descubrir el camino de salida de un paradigma que ha estado en vigor durante largas décadas, y cuyas ideas básicas todavía permanecen fuertemente arraigadas, en diverso grado, en las mentes de estudiosos y profesionales del periodismo. La alternativa a él habrá de ser fruto, necesariamente, de largos años de reflexión colectiva. En consecuencia, para evitar suscitar falsas expectativas en el lector, me apresuro desde ahora a dejar claro que me voy a limitar a argumentar por qué, a mi entender, el viejo paradigma objetivista está mal concebido y es erróneo y, en consecuencia, se encuentra en intelectualmente fracturado. El apuntar tan siquiera las bases para la construcción de una alternativa desborda, con mucho, las posibilidades de este artículo, aunque no por ello dejaré de ofrecer alguna sugerencia final al respecto.

\section{Un concepto insuficientemente definido}

En primer lugar, es oportuno notar que la mayoría de los debates sobre la teoría de la objetividad adolecen de la falta de un concepto sólido y bien definido de lo que se entiende bajo este término. No estoy con esto sugiriendo, en absoluto, que todo el problema pueda ser reducido a una cuestión de semántica. Pero sí que hay razones suficientes para pensar que muchos malentendidos proceden del uso de una noción bastante indefinida y difusa.

GAUTHIER (1993: 1) critica -en mi opinión, de una manera exagerada- la falta de definición del concepto, tal como se usa en periodismo: "Uno de los más grandes problemas en cualquier debate sobre la objetividad periodística es la naturaleza ambigua y elástica de este concepto. Rara vez nos tomamos la molestia de identificar los aspectos o elementos de la tarea informativa a la que el concepto de objetividad puede ser aplicado. En otras palabras, hay una considerable confusión sobre qué se supone que es objetivo en periodismo [...]. La idea que se rechaza no es una noción clara, fácilmente identificable, sino más bien una intuición vaga: el objeto que se ataca nunca fue definido con precisión".

DuRHAM (1998: 118), por su parte, coincide en parte con esta postura, aunque de una manera más matizada, cuando afirma que "la objetividad periodística ha sido siempre una noción resbaladiza".

No comparto la afirmación de que la noción de objetividad sea una "intuición vaga", porque algunos autores sí han propuesto buenas definiciones de ella en el pasado. Pero sí estoy de acuerdo con que, en conjunto, es una noción resbaladiza, porque ciertamente tiene un carácter elusivo, debido a varias razones que veremos más adelante. Esta ambigüedad conceptual queda bien ilustrada por las palabras de ROSEN antes citadas (1993: 48). 
Otros autores coinciden en señalar que la objetividad es "una de las grandes confusiones en periodismo" (Kovach y Rosenstiel, 2001: 72), hasta el punto de que "en el presente, el significado original de esta idea es completamente malentendido y, en conjunto, se ha perdido" (72). Finalmente, algunos otros autores, como Brent CuNNINGHAM (2003: 1), editor de la Columbia Journalism Review, transmiten la misma idea en forma de ocurrencia jocosa: "Pregunte a diez periodistas qué significa objetividad y recibirá diez respuestas diferentes".

No es extraño que el concepto de objetividad oscile entre la vaguedad y la confusión, debido, en mi opinión, a las principales razones siguientes:

1. Se trata de un asunto complejo, que -perteneciendo a la entraña del periodismoestá en relación con muchos otros y, como tal, puede ser abordado desde perspectivas muy diferentes ${ }^{2}$.

2. Con el tiempo, el término ha llegado a abarcar una gran cantidad de significados divergentes e incluso opuestos; en otras palabras, el propio concepto no ha permanecido inalterado históricamente, sino que ha cambiado diacrónicamente ${ }^{3}$.

3. El problema de la objetividad tiene un origen filosófico, lo que significa que sus ideas nucleares proceden de conceptos filosóficos profundos y difíciles - como el de verdad-, sin los cuales no puede ser plenamente entendido.

Desde este momento, voy a centrarme en la tercera de estas razones, que, a mi juicio, ha sido descuidada de forma manifiesta.

\section{Nociones recurrentes en las principales definiciones de objetividad}

A pesar de la complejidad del concepto de objetividad y de sus cambios históricos, hay una base común de nociones recurrentes que subyacen en él. Puesto que una revisión completa de tales ideas nos alejaría del objeto de este estudio, trataré ahora de sinte-

${ }^{2}$ La idea de objetividad tiene un carácter elusivo debido en parte a su estrecha relación con otros muchos asuntos mediáticos, que han contribuido a difuminar sus fronteras, convirtiéndolo así en un concepto poliédrico. De hecho, ha sido abordado desde perspectivas muy diferentes: en relación con la política; con las rutinas profesionales; con la evolución histórica del periodismo; con la dimensión económica de los medios; con los géneros y los formatos redaccionales; con sus fundamentos epistemológicos; con cuestiones de ética periodística; con su impacto en cuestiones relativas al sexo, la raza o la clase social, etc. La relación de autores que han cultivado estas y otras facetas sería interminable.

3 Para cualquier académico familiarizado con este tema, es bastante obvio que la definición de objetividad ha variado lo largo de los años (SCHUDSON, 1990; STOKER, 1995; MINDICH, 1998). El concepto original, inspirado en gran medida en los postulados de la Escuela de Sociología de Chicago -que pretendía aplicar los rigurosos métodos de las nuevas ciencias sociales al periodismo-, tiene poco que ver con otros enfoques recientes, marcados por el relativismo. No es éste el lugar apropiado para hacer una revisión de los principales hitos en la evolución histórica del concepto, sobre lo cual existen sólidos estudios (e.g., MindicH, 1998; KAPLAN, 2002). Tampoco éste es el sitio idóneo para tratar acerca de las diferencias conceptuales que existen entre diversos países, acerca de lo cual también hay disponible investigación rigurosa (e.g., DONSBACH y KLETT, 1993). Sólo se trata aquí de recordar el modo en que estos cambios, tanto en el tiempo como en el espacio, han aumentado considerablemente las dificultades para clarificar un concepto ya de por sí problemático. 
tizarlas en dos grandes categorías -epistemológica y ética-, con el fin de clarificar el significado del término "objetividad", con vistas a su uso en este artículo.

Si hay una idea que comparte la mayoría de definiciones de objetividad, ésta es, sin duda, la idea de la facticidad libre de valores, que, supuestamente, debe regir la actividad informativa. En torno a ella, gira la primera categoría. Como dice HACKETT (1984: 232), "el ideal de objetividad señala que los hechos pueden ser separados de las opiniones y de los juicios de valor, y que los periodistas pueden permanecer al margen de los acontecimientos del mundo real cuya verdad o significado transmiten a la audiencia por medio de un lenguaje neutral y de técnicas informativas adecuadas". En consecuencia, "la inclusión del valor en el relato de los hechos -amplía REESE (1990: 393-394)- amenaza la objetividad, y el periodista que tiene valores sólidos amenaza el buen funcionamiento del sistema".

En la misma línea, el concepto de objetividad es definido habitualmente como lo opuesto a la visión sesgada o partidista: "La mayoría de las definiciones en lengua corriente consideran el sesgo informativo como la intrusión de la opinión subjetiva por parte del periodista o de la organización informativa en lo que se supone que es un relato factual. Así MACLEAN $(1981,56)$ sugiere que 'cuando una noticia no distingue claramente entre las interpretaciones de su autor y los hechos de los que informa, se trata de una noticia tendenciosa o sesgada"" (НАCKETT, 1984: 230). En resumen, esta idea de que los periodistas deben "mantenerse al margen", neutrales, cuando "transmiten los hechos", prohibiéndose a sí mismos que sus valores y opiniones "interfieran" en las tareas informativas, está inevitablemente presente, de una manera u otra, cuando se habla de objetividad.

Junto a este primer sentido, que podríamos denominar "epistemológico", hay también un conjunto de ideas sobre la objetividad centradas, principalmente, en el lado "ético" de la cuestión. Me refiero a todo aquello relacionado con lo que se llama "balance", "equidad" o "no manipulación". Por supuesto, no es posible establecer una línea divisoria tajante entre la dimensión epistemológica y la ética, pues ambas están relacionadas (como también lo están la inteligencia y la voluntad de los seres humanos). De hecho, ambas tienden a mezclarse con frecuencia. Pero, mientras en el primer caso, se pone el énfasis en cómo los periodistas pueden conocer sin interpretar -es decir, eliminando de raíz sus opiniones y valores-, con independencia de sus actitudes e intereses, el enfoque ético, en cambio, se centra en su integridad moral. Así, en teoría, uno podría ser objetivo en cuanto al modo en que conoce "los hechos", pero no querer usar esta capacidad por falta de ética, por ejemplo, haciendo a propósito juicios injustos y manipulando las noticias según sus intereses.

Como ya se ha dicho, la objetividad planteada primariamente como una cuestión ética es entendida en términos de justicia y equidad. De acuerdo con el principio de equidad, el fin de la buena información es presentar visiones múltiples, o incluso opuestas, sobre un tema, como si todas ellas fueran igualmente válidas, es decir, sin conceder el menor privilegio a ninguna de ellas. Desde esta perspectiva, "presentar una historia objetivamente implica escribir y organizar el material para que no exprese o sugiera una preferencia mayor por un conjunto de valores que sobre otro" (KLAIDMAN y BEAUCHAMP, 1987: 46). 
Estos dos enfoques principales de la cuestión no solo están relacionadas conceptualmente, sino también históricamente, en cuanto a su evolución. Cuanto más se ha criticado, por insostenible, la concepción de la objetividad "más epistemológica", más se ha desarrollado y prevalecido el enfoque "más ético". "A causa de estas críticas, la gradual evolución de la norma de objetividad periodística ha supuesto un alejamiento del énfasis en la 'neutralidad' y un poner en primer plano las ideas de 'exactitud', 'imparcialidad' y 'equidad'” (DuRHAM, 1998: 119; cf. también REESE, 1990: 393).

Como prueba de esta estrecha conexión, en los últimos veinticinco años, aproximadamente, muchos periodistas empezaron a decir que ya no "creían" más en la objetividad, sino solo en la honradez y en la integridad moral. De esta manera, reducían así el problema del conocimiento a una cuestión de valores éticos (entendidos, además, en la mayor parte de los casos, en clave relativista). Un periodista con larga experiencia como Martin BELL (1998: 102) nos proporciona un buen ejemplo de este cambio, y también de la confusión antes mencionada, cuando escribe: "Permítanme empezar con una herejía. Yo fui formado en la tradición del periodismo objetivo y desapasionado. Una vez creí en él. Ya no creo más en él. [...] En lo que todavía creo, y a lo que me aferro más que nunca, es en la equidad e imparcialidad y en una atención escrupulosa a los hechos y en la determinación a prestar atención a los portavoces impopulares de las causas desfavorecidas".

Dicho esto, debo aclarar ahora que, a lo largo de este texto, me voy a ocupar principalmente del primer modo de entender la objetividad, que es el que considero más fundamental. En primer lugar, porque, históricamente, ha tenido mucho mayor peso que su posterior reconceptualización ética. En segundo lugar, porque esta última está esencialmente arraigada en el terreno epistemológico de la facticidad libre de valor, que -como afirma REESE con acierto- todavía "permanece firmemente atrincherada" en nuestras mentes.

\section{Premisas filosóficas subyacentes en la Teoría de la Objetividad \\ 4.1. El positivismo como origen de la teoría de la objetividad y su vigor contem- poráneo}

Una vez revisados brevemente los aspectos centrales de la problemática definición de objetividad, tratemos ahora de lograr un mejor entendimiento de los asuntos en juego, profundizando en sus fundamentos filosóficos, con frecuencia, ignorados o preteridos, sobre todo, por un gran número de periodistas.

En primer lugar, creo oportuno comenzar por mostrar que la teoría de la objetividad no es, en modo alguno, original o exclusiva del periodismo. Más bien se trata de una variante de la teoría de la objetividad científica, que deriva de la filosofía empirista y de su heredero, el positivismo. En efecto, la fuente de la idea de objetividad se remonta al pensamiento de Augusto ComTe (1798-1857), que a su vez se basa, en parte, en el de Hume (1711-1776), desarrollado y extendido posteriormente, a lo largo de los siglos XIX y XX, por sus muchos epígonos, como los miembros del Círculo de Viena. El gran éxito que experimentaron las ciencias naturales a lo largo siglo XIX alentó el intento positivista de tratar de aplicar el método empírico -cuyos brillantes resultados eran obvios- a las ciencias sociales y a las humanidades, basándose en la presuposición de que éste era el único método válido. 
Esta sobrevaloración del método empírico como único método científico posible acarreó la aceptación de las premisas medulares del positivismo y su posterior extensión a otros campos. Entre tales premisas, la más importante es la dicotomía que opone los llamados "juicios de hecho" a los "juicios de valor" (una distinción adoptada posteriormente en periodismo casi al pie de la letra, como veremos más adelante). Como se sabe, la tradición positivista sostiene como un dogma que solo las afirmaciones referidas a "hechos" deben ser consideradas "objetivas" y, por tanto, válidas; mientras que los juicios referidos a cuestiones humanas, en las cuales los valores juegan un papel decisivo, son "subjetivos", es decir, mera expresión de preferencias empíricamente inverificables y, por tanto, sin ninguna validez racional, o incluso carentes de todo significado (e.g., HEMPEL, 1965). De acuerdo, pues, con el positivismo, verdad se convierte en equivalente de objetividad y, para lograrla, uno debe "atenerse a los hechos", "dejar que los hechos hablen por sí mismos", sin ninguna interferencia por parte del sujeto que los conoce y transmite a los demás.

No es preciso recurrir a la historia para poder afirmar que, desde el siglo XIX hasta el presente, la doctrina positivista ha mantenido la mayor parte de su vigor (e.g., MuÑOZ TORRES, 2002). Una prueba de esto es cómo surge el problema de la objetividad en muchos terrenos académicos y cómo todavía crea un alto grado de controversia y debate. A modo de ejemplo: en el campo de la sociología del conocimiento, "es probable -sostiene LAMO DE ESPINOSA (1984: 83)- que la mayor parte de los trabajos de las últimas décadas se hayan generado alrededor de la problemática de cómo alcanzar la objetividad en las ciencias sociales".

La influencia del pensamiento positivista en el campo de los medios ha sido también fuerte y omnipresente desde el siglo XIX, hasta el punto de que ha impregnado completamente sus principios y sus prácticas profesionales. Así, por ejemplo, un correlato de la dicotomía hecho-valor se encuentra en el contraste tajante entre noticias ("no valorativas") y comentarios ("valorativos"). Ya en 1855, Samuel Bowles distinguía entre "noticias de hechos" y "noticias de opiniones" en un editorial escrito para su periódico, el Springfield Republican (RoshCo, 1975: 39). En definitiva, como afirma SCHUDSON (1978: 4-5), "la creencia en la objetividad es una fe en los 'hechos', una desconfianza en los 'valores' y el compromiso de separar ambos".

De manera similar, la idea de que la objetividad exige que los enunciados científicos excluyan los juicios de valor (según la teoría weberiana de la Wertfreiheit o ausencia de valores) encuentra su equivalente directo en el deber de neutralidad, o también en la carencia de sesgo, al informar, tal como hemos visto en el apartado 3.

Finalmente, este dualismo llegó, con el tiempo, a ser tan aceptado y estar tan extendido entre periodistas y académicos que incluso fue acuñado en forma de frase hecha, muy popular, todavía citada con frecuencia: "los hechos son sagrados, las opiniones son libres", a la cual se apela como si se tratara de un principio axiomático indiscutible y cuya solvencia no resiste un análisis mínimamente riguroso (e.g., GonZÁlez Gaitano, 1989: 17).

\subsection{La dicotomía hecho-valor: una distinción tan clara como falsa}

Si tuviéramos que sintetizar el meollo de la teoría de la objetividad, éste consistiría, a mi juicio, en su postulado principal: la mencionada dicotomía hecho-valor, según la 
cual el verdadero conocimiento se reduce a objetividad y la subjetividad se considera refractaria a toda verdad. Como el célebre filósofo Hilary PutnAm (2002: 1) advierte acertadamente: "La idea de que 'los juicios de valor son subjetivos' es un ápice de filosofía que ha llevado gradualmente a ser aceptado por mucha gente como si fuera de sentido común. [...] [Asimismo] los 'enunciados fácticos' son susceptibles de ser 'objetivamente verdaderos' y susceptibles también de ser 'justificados objetivamente', mientras que los juicios de valor no admiten verdad objetiva ni justificación objetiva".

Sin duda, el éxito de este dualismo entre las personas sencillas, que no son filósofos, se debe a su gran claridad; pero -como debería ser obvio- ésta, per se, no lo hace válido. Más bien sucede lo contrario. Veamos ahora por qué se trata de una simplificación insostenible, que no puede ser defendida ni tan siquiera como una distinción analítica. En lugar de considerarla como un dogma que constituye la piedra angular del conocimiento, deberíamos no dar por supuesta su validez, sino someterla a análisis crítico. Aunque éste es un problema filosófico complejo, que ha sido desarrollado de maneras muy diversas por muchos autores, intentaré abordarlo, a continuación, de una forma concisa, sobre la base de los tres argumentos siguientes:

1. La imposibilidad de una percepción pura de hechos desprovista de conceptos previos.

2. La imposibilidad de seleccionar hechos sin valores.

3. Las contradicciones inherentes a la pretensión positivista.

\subsubsection{Por qué es imposible una percepción pura de hechos desprovista de con- ceptos previos}

Empecemos por considerar que no existe una pura percepción de hechos, despojada de cualquier concepto previo. La razón es clara: para poder identificar algo como hecho, necesitamos un concepto previo de "hecho". ¿Qué debe entenderse bajo el término "hecho"? O bien, como se pregunta Putnam (1981: 163), "¿desde qué punto de vista se usa la palabra 'hecho'?".

La ideología positivista no se preocupa por la respuesta a estas preguntas: la da por supuesta. Concibe a los científicos o investigadores como meros observadores que se limitan a "recolectar hechos", como si fueran entomólogos que capturaran insectos. Estos observadores se comportarían — sostiene el positivismo- como si carecieran de un conjunto previo de conceptos teóricos y de valores prácticos. Pero, si esto fuera así, entonces no sabrían qué buscar. Alasdair MACINTYRE pone este ejemplo para mostrar lo erróneo de esta postura: "El observador del siglo XX mira al cielo nocturno y ve estrellas y planetas; algunos observadores anteriores, en lugar de esto vieron grietas en una esfera, a través de las cuales podía observarse la luz del más allá. Lo que cada observador cree percibir se identifica y tiene que ser identificado por conceptos cargados de teoría. Los perceptores sin conceptos, como vino a decir Kant, están ciegos" (MACINTYRE, [1981] 1987: 196; la cursiva es mía).

En efecto, los hechos no son puros datos pre-conceptuales, sino interpretaciones de percepciones sensoriales ${ }^{4}$, identificadas por medio de nociones cargadas de teoría.

${ }^{4}$ Me parece obvio que los datos sensoriales son previos a cualquier actividad de conocimiento intelectual. Por tanto, no pretendo decir aquí que toda percepción requiera una base con- 
Por tanto, cualquier descripción de la realidad -incluso aunque trate de ser la representación más exacta de ella- presupone un buen número de conceptos teóricos y de juicios derivados de ellos. Conocer implica, por definición, hacer juicios, y esto inexorablemente equivale a combinar datos sensoriales con conceptos, tanto teóricos como morales.

Como MACINTYRE observa con perspicacia: "Si toda nuestra experiencia tuviera que caracterizarse en términos de este desnudo tipo de descripción sensorial [...], nos enfrentaríamos no solo a un mundo sin interpretar, sino ininterpretable, a un mundo no simplemente no abarcado aún por la teoría, sino a un mundo que nunca podría ser abarcado por la teoría. Un mundo de texturas, formas, olores, sensaciones, sonidos y nada más, no propicia preguntas y no proporciona ningún fundamento para respuestas" (MACINTYRE, [1981] 1987: 107). Y, para dejar claro que la experiencia no puede ser el único criterio de verdad, continúa más adelante: "El concepto empirista pretendió discriminar los elementos básicos con que se construye nuestro conocimiento y sobre los que se funda; creencias y teorías se validan o no, dependiendo del veredicto de los elementos básicos de la experiencia. Pero las observaciones del científico naturalista no son nunca básicas en este sentido. En efecto, sometemos las hipótesis a la prueba de la observación, pero nuestras observaciones pueden cuestionarse siempre. La creencia de que Júpiter tiene nueve lunas se prueba mirando a través de un telescopio, pero esta misma observación tiene que legitimarse por medio de las teorías de la óptica geométrica. Es tan precisa una teoría que apoye la observación como la observación lo es para la teoría" (MACINTYRE, [1981] 1987: 108; la cursiva es mía).

En definitiva, los "hechos brutos" carecen completamente de sentido si no están conectados -mediante la subjetividad de la persona- con conceptos y nociones que hacen posible su interpretación. De ahí que el conocimiento factual no pueda ser disociado de la percepción subjetiva y, en consecuencia, de la capacidad humana de juzgar. En las últimas décadas, esta idea se ha ido abriendo camino lentamente también entre los estudiosos de los medios. Hay buenos ejemplos al respecto. Tomemos solo uno, en aras de la brevedad. Philip MEYER (1993: 33-34), padre del llamado "periodismo de precisión", escribe: "Los datos brutos nunca bastan por sí mismos. Para que sean útiles e inteligibles han de ser procesados, conceptualizados, integrados en algún tipo de esquema. Hay que insertar el dato material en algún marco de referencia mental que ayude a su interpretación y comprensibilidad".

\subsubsection{Por qué es imposible seleccionar hechos sin valores}

Supongamos ahora, per hypothesis, que los hechos pudieran ser conocidos por un sujeto carente de cualquier concepto o valor previos. Incluso en ese caso, tratar de adquirir un conocimiento objetivo sobre la realidad implicaría necesariamente elegir entre unos hechos y otros. El mundo empírico nos ofrece infinitos datos, cuyo conocimiento -por pequeño que sea- no puede abarcar. Por eso, es inevitable tenerlos que seleccionar de acuerdo con los valores del sujeto. Y, entre estos valores, el principal

ceptual previa o que sea una proyección del yo, como algunas formas de constructivismo sostienen. 
es la relevancia con vistas a un fin. Así, cualquier investigador científico debe necesariamente filtrar hechos y datos en tanto en cuanto son considerados relevantes para el éxito de los fines de su investigación. Por consiguiente, los fines abren inexorablemente el camino a los valores, aunque de manera implícita la mayor parte de las veces.

No es extraño entonces que los "hechos" relevantes para un sociólogo no lo sean, en absoluto, para un químico, o viceversa. Solo aquellos que ya comparten un marco conceptual previo saben cómo identificar lo que será tomado como "hecho" dentro de él. Más aún, incluso el mismo "hecho" puede ser considerado relevante o no para la misma persona, según el punto de vista desde el cual es observado y según los objetivos que se tienen en mente. En suma, cada selección está, esencialmente, cargada de valores y nunca puede estar libre de ellos, es decir, entraña siempre un conjunto de valores explícitos o implícitos aceptados subjetivamente por la persona que hace la selección. En consecuencia, la supuesta neutralidad del conocimiento en general, o de la ciencia en particular, es solo un espejismo.

Trasladando esto al caso del periodismo, es obvio que existe un continuo proceso de selección de "hechos", en función de su supuesta importancia para la sociedad. Basta observar cómo los distintos medios seleccionan y presentan los acontecimientos noticiosos de manera diferente, o incluso totalmente opuesta, para darse cuenta de que la selección no valorativa de éstos es completamente imposible. Si fuera posible, entonces bastaría con transmitir "los hechos" objetivamente a través de un solo medio, mientras que los restantes medios se centrarían en su interpretación y valoración. Pero la interpretación y valoración de los hechos son siempre inherentes a la misma selección y al modo en que ésta se presenta. Por tanto, una situación tan absurda como ésta es imposible de concebir hasta como hipótesis.

\subsubsection{Las contradicciones inherentes a la teoría de la objetividad}

Para concluir este apartado, trataré ahora de mostrar brevemente que la pretensión positivista sobre la objetividad, tanto en la ciencia como en el conocimiento general, no solo es imposible, sino una contradicción en sí misma. Dicho más claramente: sostener que el conocimiento, para ser objetivo y verdadero, debe excluir cualquier juicio valor es incurrir en contradicción.

En efecto. Tan pronto como uno dice: "el conocimiento, para ser objetivo, debe estar libre de valores", está en ese mismo momento haciendo un juicio de valor implícito, que considera la neutralidad como el valor primordial. En otras palabras, el positivismo sostiene que solo el conocimiento libre de valores es auténtico conocimiento $\mathrm{y}$, por tanto, que los valores deben ser erradicados de él. O también que se debe perseguir el conocimiento factual, porque es mucho mejor que el valorativo. Si consideramos estas afirmaciones con detenimiento, nos daremos cuenta inmediatamente de que no son, en absoluto, enunciados factuales, puesto que ni se basan en hechos, ni proceden de la experiencia, ni tampoco pueden ser verificados empíricamente. Más bien, se trata de juicios de valor encubiertos, presentados como si fueran axiomas evidentes, cuando no lo son.

En el ámbito de los medios, el lenguaje es también bastante revelador. Los periodistas deben ser objetivos, porque los juicios de valor, en tanto en cuanto distorsionan 
la realidad, son considerados malos. En lugar de eso, lo adecuado es que los periodistas traten de ser neutrales, porque introducir las propias opiniones en una noticia es "arriesgado" (RICH, 1997: 204), y que mantengan igual distancia respecto a las fuentes divergentes. Como el caso de la ciencia, quienes exigen que los valores sean excluidos del periodismo lo hacen porque piensan, paradójicamente, que éste es el mejor modo de conocer y de informar. En consecuencia, como vemos, los valores son inseparables de las premisas básicas del positivismo, están implícitos necesariamente en su mismo origen.

De los argumentos brevemente expuestos en los tres apartados precedentes, se puede extraer una conclusión clara: la dicotomía hecho-valor -a pesar de presentarse a sí misma como clara e improblemática, como si fuera un axioma- es falsa y racionalmente insostenible. Establece hábilmente un falso dualismo entre hechos y valores, como si pudieran separarse fácilmente unos de otros, ignorando así que están entremezclados en la realidad, la cual es bastante más compleja que un esquema tan fácil y cómodo como equivocado.

\section{EI marco conceptual positivista y sus preguntas mal concebidas}

Aunque la dicotomía hecho-valor es errónea, como acabamos de ver, esto explica por qué la teoría de la objetividad se basa sobre una pregunta mal concebida, que origina debates inconcluyentes. Ha llegado el momento de tratar este punto arriesgado. Lo que pretendo hacer en este apartado es argumentar que, subyacente a la teoría de la objetividad, existe una gran confusión de objetividad con verdad y de subjetividad con carencia de ella.

\subsection{El intento de sustituir verdad por objetividad}

En el terreno de los medios, durante muchas décadas, el concepto de objetividad ha logrado reemplazar casi completamente al de verdad (MUÑOz-TORRES, 2007), objeto frecuente de prejuicios y errores conceptuales. La lógica que subyace a esta sustitución radica en la asunción de que la verdad es un concepto vago y elusivo, inalcanzable para los seres humanos (e.g., VÁzQuEZ, 1986, 9). Ahora bien, si se acepta la premisa "la verdad es inalcanzable" (es decir, se toma como verdadera, incurriendo así en contradicción ${ }^{5}$ ), entonces la única opción posible es sustituir el concepto de verdad por otros como los de objetividad o incluso aquellos que se derivan de él, como los de exactitud, equidad o imparcialidad. Esto es lo que ha ocurrido históricamente, como hemos visto, de pasada, en el apartado 3.

La cuestión clave ahora es: ¿puede la noción de objetividad reemplazar con éxito al concepto de verdad? Si es así, ¿con qué implicaciones? A continuación, veremos que

\footnotetext{
5 “'Cuando se dice que 'la verdad es inalcanzable', se presupone eo ipso que esta misma afirmación es en sí misma verdadera, es decir, que explica plenamente la capacidad humana de conocer. De ahí que nadie pueda afirmar que la verdad no existe sin considerar su propia afirmación verdadera. De la misma manera, la postura de que la verdad es inalcanzable, a causa de su supuesto carácter vago y elusivo, se basa necesariamente en un concepto implícito de verdad, aceptado inconscientemente. De lo contrario, carecería de sentido" (MUÑOZ-TORRES, 2007: 236).
} 
esto no es posible sin distorsionar el significado del concepto de verdad y, en el fondo, sin negarlo.

Parece obvio que cualquier acto de conocimiento implica, por definición, una relación entre dos términos: entre un sujeto y un objeto. Si esto es así, entonces también es evidente que cualquier acto de conocimiento tiene constitutivamente una doble dimensión: la relativa al sujeto que conoce y la que se refiere al objeto conocido ${ }^{6}$. Sin alguno de estos dos términos, no puede darse el conocimiento en absoluto, de manera análoga a como sucede con otros tipos de relación. Tomemos, por ejemplo, la relación biológica de maternidad: es evidente que nadie puede ser madre sin tener hijos, y viceversa. Estableciendo un paralelismo entre conocimiento y maternidad, la pregunta acerca de cómo nosotros, siendo sujetos, podemos conocer objetivamente, equivaldría a preguntar cómo una mujer puede ser madre biológica sin tener hijos. Ambas son preguntas mal concebidas, que intentan en vano desafiar las leyes de la lógica.

Trasladando el ejemplo al objeto de nuestro interés, si el conocimiento, por definición, siempre necesita un sujeto y un objeto, ¿qué sentido tiene intentar excluir al sujeto a cualquier precio, como si el objeto pudiera ser conocido sin sujeto? A la luz de este argumento, la pretensión central de la teoría de la objetividad periodística parece absurda. De manera análoga, uno puede preguntar: ¿pueden los periodistas excluirse a sí mismos cuando conocen lo que ocurre en la sociedad, poniendo al margen sus propias ideas y opiniones? ¿Pueden realmente dejar fuera cualquier juicio valor o interpretación, permitiendo que los hechos "hablen" por sí mismos? Obviamente no. Dejar que los hechos "hablen" por sí mismos es una metáfora, tomada erróneamente al pie de la letra, que nunca debería haber llegado tan lejos como lo ha hecho. Ningún periodista puede conocer sin hacer juicios y éstos, como hemos visto, requieren necesariamente de un conjunto previo de ideas y valores. En consecuencia, la pretensión positivista de auto-exclusión en el conocimiento, ni es conceptualmente posible, ni por consiguiente- es viable en la práctica.

En ocasiones, llega a ser tan obvio que el concepto de objetividad es profundamente erróneo, que muchos académicos y periodistas tratan de desviar las críticas añadiendo algunos matices a la cuestión. Así, se puede escuchar con frecuencia que, aunque la "plena" objetividad es imposible, los periodistas deben esforzarse "al máximo" para intentar conseguir el "más alto grado posible" de ella. Para mí, se trata de un "buen intento" (como dicen los anglosajones), pero, en el fondo, vano. La razón es clara: no estamos hablando de algo que es posible de distintas maneras o en distintos grados, sino de una imposibilidad absoluta. Nadie piensa que la miopía pueda eliminarse por un acto de voluntad. De manera similar, ninguna determinación de la voluntad -por muy fuerte que sea- puede erradicar la subjetividad del conocimiento. Querámoslo o no, todos conocemos a través de nuestra miopía mental, o sea, de un modo limitado, precario y subjetivo, aunque no refractario a la verdad.

6 El término "subjetivo" tiene significados diversos. Desde la perspectiva epistemológica, adoptada aquí, "subjetivo" significa relativo a la persona que conoce y, por tanto, limitado, falible y precario (aunque no imposible). Pero, desde otra perspectiva (ontológica), también puede significar "arbitrario", "caprichoso", "gratuito", es decir, falto de correspondencia con la realidad. Aquí uso el término con el primer significado. 
Yendo un paso más allá, de las consideraciones precedentes se sigue que la verdad, como propiedad esencial del conocimiento efectivo, no puede ser "reducida" ni a su dimensión objetiva, ni subjetiva. Ambas están siempre inevitablemente presentes. Sin cualquiera de ellas, el conocimiento sería imposible, bien por la falta de un objeto que conocer, bien por la falta de un sujeto individual que conoce. En conclusión, sostener que el conocimiento es verdadero en la medida en que es objetivo (por exclusión de su dimensión subjetiva) es una grosera simplificación insostenible.

Finalmente, mientras que nadie sensato se plantea preguntas absurdas, como la de cómo ser madre sin tener hijos -por ser lógicamente inadmisible-, el positivismo, en cambio, ha logrado hacer esto con el conocimiento de una manera extremadamente exitosa históricamente. Hasta tal punto ha sido esto así, que lo que originalmente era una discusión filosófica de unos pocos académicos, se abrió paso y se extendió, con el tiempo, al hombre de la calle. De esta forma, el positivismo ha conseguido establecer un marco conceptual erróneamente dualista, que vicia la entera cuestión de la objetividad desde su mismo planteamiento. Pese a ello, esta visión dicotómica del conocimiento y de la verdad está tan profundamente arraigada en nuestra mentalidad contemporánea, que es muy difícil sustraerse a ella, como veremos a continuación.

\subsection{Un movimiento pendular desde la objetividad: el relativismo}

Una vez que el marco conceptual positivista está arraigado en nuestras mentes, cabe esperar un movimiento pendular, a partir del rechazo de la objetividad, hacia lo que parece ser su opuesto, la subjetividad. Esto es exactamente lo que ha ocurrido en la práctica profesional del periodismo y, especialmente, en la reflexión sobre los medios. Como se dijo en la Introducción, durante las últimas décadas, los fundamentos filosóficos de la objetividad han sido reevaluados y, finalmente, rechazados. Éste rechazo de la objetividad ha dado lugar, como reacción, al nacimiento de muchas teorías y corrientes de pensamiento que han puesto todo el énfasis en la subjetividad (una exposición de las principales, en RYAN, 2001).

Como Ryan (2001: 6) indica, "el periodismo no ha escapado de la influencia de los relativistas (p.ej., Altheide, 1976; HARding, 1991; Longino, 1990; ShoemaKer y REESE, 1991), que argumentan que los absolutos no existen en el conocimiento, en la moral, o en los valores". El relativismo ha florecido, ciertamente, no solo en el ámbito de los medios, sino en la mayor parte de los terrenos académicos. Su principio más básico -que los criterios de evaluación de verdad y racionalidad son relativos al sujeto individual y a su pertenencia a una cultura, una clase social, un sexo, una raza, etc.es más que familiar para todos nosotros, porque está omnipresente de manera implícita o explícita por todas partes.

Centrándonos ahora en los medios, el relativismo ha ido tomando el lugar de la teoría de la objetividad, a medida que ésta ha ido declinando. Aunque el pensamiento relativista no es objeto central de este artículo y merecería mucha más atención, ofreceré ahora una rápida reflexión sobre una de sus manifestaciones: el principio de imparcialidad, antes explicado, que guarda también relación con las premisas objetivistas, en la medida en que éstas exigen que los periodistas se abstengan de favorecer, respecto a un asunto controvertido, una de las versiones contendientes sobre 
las demás. Pero, en este caso, se pone el énfasis sobre el valor idéntico que se da a todas las opiniones y juicios de valor. Como DuRHAM (1998: 124) adecuadamente observa: "El periodismo ha abrazado esta clase de relativismo como una parte integral de sus prácticas, puesto que los periodistas son entrenados para buscar visiones opuestas sobre un asunto dado como prueba de su objetividad al informar".

Parece bastante claro que ofrecer visiones plurales sobre un tema puede ser enriquecedor y valioso, especialmente, si no hay pruebas disponibles sobre cuál es la verdadera, o cuando se trata solo de mera cuestión de preferencias. Sin embargo, no es menos cierto que el principio de presentar siempre las visiones opuestas sobre algo como igualmente válidas, equivale a afirmar implícitamente que todas las opiniones poseen el mismo valor de verdad. Y, si todas ellas son igualmente válidas, en el fondo, ninguna de ella es válida en absoluto o, dicho de otro modo, todas ellas son refractarias a la verdad ${ }^{7}$ (como quiere el relativismo, al sostener que la verdad es inalcanzable).

Dicho por medio de un ejemplo, HARDING se pregunta: “¿Qué significaría afirmar que no hay criterios razonables que puedan o pudieran en principio ser hallados para discernir entre la pretensión de una cultura que sostiene que la tierra es plana y la de otra cultura que sostiene que la tierra es redonda?" (HARDING, 1991: 139, cit. por DuRHAM, 1998: 126).

Rosen (1993: 49) proporciona otro ejemplo, muy ilustrativo, tomado de la práctica periodística: "Por una parte, el Tobacco Institute dice que fumar es bueno y que hasta incluso mejora tu salud, pero por otra parte la Sociedad Americana de Cáncer dice que fumar te matará... el periodismo nos muestra que, con frecuencia, el equilibrio [equidistante] es una huida de la verdad más que una avenida hacia ella".

Algo similar puede decirse sobre temas en los que la presencia de valores morales es más patente. Es ridículo sugerir que puede haber neutralidad legítima en cuestiones de aborto, racismo, terrorismo, abuso infantil, trata de mujeres, tráfico de drogas, etc.

\subsection{Objectivismo y relativismo, ¿dos caras de la misma moneda?}

Tras esta rápida mención al relativismo en periodismo, intentaré establecer ahora algunas conexiones con la línea de razonamiento precedente. Si prestamos debida atención al relativismo, podemos fácilmente concluir que, lejos de ser lo opuesto del

\footnotetext{
${ }^{7}$ En relación con este asunto, en otro lugar he argumentado lo siguiente: "Es obvio que no opinión es la expresión de un juicio fruto de una evaluación y, naturalmente, es subjetiva. Sin embargo, no hay ninguna razón para suponer que subjetivo es sinónimo de falso. Es verdad que algunas opiniones son expresión de sentimientos o de gustos y manías que son imposibles de verificar para otra persona, pero la conclusión lógica no es el principio empirista de que la opinión, por definición, es completamente ajena a la verdad (e.g., HEMPEL, 1965). Como todos sabemos, hay opiniones insensatas, arbitrarias, no basadas en pruebas sólidas, ni en razonamientos cabales; y hay también opiniones sólidas, basadas en razonamientos convincentes o en pruebas empíricas irrefutables. Pienso que el problema radica en lo engañoso de las apariencias. Las opiniones se expresan normalmente con un lenguaje más 'personal', colorista, que tiende a mezclar pensamientos, actitudes y sentimientos. Sin embargo, tales opiniones pueden ser más verdaderas que los análisis que se presentan de un modo directo, impersonal, pero basados en datos falsos o en asociaciones arbitrarias" (MUÑOZ-TORRES, 2007: 240).
} 
objetivismo, como parece a primera vista, está estrechamente relacionado con él. La razón es bastante clara: objetivismo y relativismo suelen ir unidos como dos caras de la misma moneda, porque ambos forman parte del marco conceptual positivista.

Tomando en préstamo el modo en que PUTNAM (1981: 143) explica esta idea, cabe afirmar que somos demasiado positivistas con respecto a algunas materias, como la física, porque las vemos "como la Única Teoría Verdadera, y no simplemente como una descripción racionalmente aceptable adecuada para ciertos problemas y propósitos". Así, tomar la prueba empírica como el último criterio de verdad, nos hace ser "subjetivistas respecto a descripciones que no podemos 'reducir' a la física" (1981: 143). En consecuencia, si lográramos ser menos positivistas en física, entonces, a mi juicio, también llegaríamos a ser menos relativistas acerca de otros temas, como la ética, la estética o los asuntos de la vida diaria.

Confrontando ahora la relación entre objetivismo y relativismo con la cuestión de la verdad -que es la propiedad esencial del conocimiento efectivo-, encontraremos claros paralelismos en ambos casos. El objetivismo identifica verdad con objeto conocido -entendido éste como un mero "hecho"- en un intento imposible de desembarazarse del sujeto. El relativismo, por su parte, adopta la posición opuesta, tratando de "reducir" la verdad a mera percepción subjetiva, en una tentativa igualmente vana de hacer a la realidad dependiente de la subjetividad individual o colectiva.

Si los positivistas fueran coherentes desde el punto de vista lógico, acabarían por reconocer que la objetividad como criterio principal para determinar la validez de nuestro conocimiento es, en sí mismo, un criterio errado (como hemos visto en los apartados precedentes). Más aún, el positivismo no solo es incapaz de probar ninguno de sus presupuestos originarios ${ }^{8}$, sino que -lo que es peor- incurre en contradicción, cuando sostiene que la experiencia es la única fuente válida de conocimiento (como también hemos visto, esta afirmación está muy lejos de ser un juicio de hecho y, además, no puede ser probada mediante la experiencia). Además, deteriora severamente el mismo concepto de conocimiento, al tratar de "reducirlo" a su objeto, mediante la ignorancia del sujeto. Algo similar puede decirse del relativismo, cuando sostiene que cada individuo o grupo establece los criterios de lo que es verdadero o falso. Si esto fuera así, entonces podríamos decidir si esta misma premisa básica es verdadera o falsa, lo que hace que la propia afirmación se autorrefute, algo devastador para cualquier doctrina.

En definitiva, tanto el positivismo como el relativismo acaban por arribar al mismo callejón sin salida, a saber, la negación del concepto mismo de verdad, en el sentido realista de adecuación entre la mente y la cosa, tal como se viene entendiendo durante siglos, desde Aristóteles. Esto es consecuencia directa, a mi juicio, de haber rechazado las que, una vez, fueron evidencias acerca del conocimiento y de haber planteado preguntas mal concebidas sobre de él. Pero, probablemente, lo peor de todo es que el positivismo ha establecido de esta manera un marco conceptual en el que nuestras

8 Por "presupuestos originarios", también me refiero, por ejemplo, a los siguientes: que el hombre pueda conocer sólo hechos, separados de conceptos e ideas, y transmitir "los hechos" aislados de ellos; y que la verdad sea inalcanzable y, por tanto, es preciso perseguir la objetividad en su lugar. 
mentes parecen estar atrapadas desde, al menos, los dos últimos siglos. En otras palabras, una vez que las premisas básicas del positivismo son aceptadas, queda obturado el camino de salida de su dualista concepción del conocimiento. No es extraño, por tanto, que la controversia sobre la objetividad en periodismo se haya convertido en interminable e inconcluyente, y oscile con demasiada frecuencia de un extremo de la dicotomía al otro.

\section{4. ¿Puede haber una vía media entre positivismo y relativismo?}

Llegados a este punto, me parece que hay algunas cuestiones que vienen inmediatamente a la mente. ¿Hay algún modo de superar el esquema conceptual al que me vengo refiriendo? ¿Puede haber una vía media entre positivismo y relativismo? A continuación, intentaré ofrecer una breve respuesta a estas dos cuestiones, estrechamente relacionadas.

Respecto a la primera, el único modo de superar el marco conceptual positivista es, en mi opinión, simplemente abandonarlo. Esto exige el hercúleo esfuerzo por rehabilitar las antiguas evidencias sobre el conocimiento, negadas por el positivismo. Si continuamos aceptando los falsos dilemas subyacentes en este marco conceptual, que nos impelen a elegir entre sujeto y objeto, razón y experiencia, no creo que haya forma de zanjar definitivamente las preguntas falaces sobre la objetividad, que seguirá siendo una cuestión aporética. Esto nos conduce a responder a la segunda pregunta. A mi juicio, la vía media entre positivismo y relativismo es el realismo clásico que emana de Aristóteles (en ocasiones, tomado por error como "empirismo ingenuo").

Obviamente, no es éste el lugar idóneo para exponer la teoría realista de la verdad, ni menos aún cómo podría ser aplicable en periodismo, porque es asunto que merece mucha atención y, además, cae fuera del objeto de este artículo. Además, esta teoría tampoco está completamente libre de problemas y, por eso mismo, queda fuera del alcance de este estudio.

Como se ha sugerido antes, el concepto realista de verdad se funda precisamente en la evidencia ignorada por el positivismo, a saber: que el conocimiento es, por definición, una relación de dos términos, de la cual surge la verdad, entendida como correspondencia entre la mente del sujeto y el objeto que tiene ante sí. Obsérvese que "subjetivo" significa aquí "relativo a la persona que conoce" y, por consiguiente, limitado, falible y precario, aunque no imposible. Todo conocimiento es, ciertamente, limitado, porque los seres humanos no somos capaces de alcanzar un conocimiento absolutamente perfecto e infalible de la realidad. Pero de ahí no se sigue, en modo alguno, que no podamos conocer en absoluto; es decir, que nuestro conocimiento no pueda alcanzar "cierta" verdad sobre las cosas, de manera análoga a como ser miope no equivale a ser ciego ${ }^{9}$.

9 En otro lugar he escrito: "La aspiración del ser humano a la verdad total puede, a mi parecer ser tan equivocada y dañina como su opuesta, es decir, como aquella que niega cualquier posibilidad de alcanzar la verdad. De hecho, concebir la verdad como algo vago, elusivo o divino puede tener, a la postre, consecuencias similares a aquellas que se derivan de la postura contraria (es decir, aquella que niega abiertamente cualquier posibilidad de verdad para el hombre). En ambos casos -en uno por exceso, en el otro por defecto-, la capacidad humana 
Con otras palabras: que el conocimiento sea subjetivo no necesariamente implica que sea "arbitrario", "caprichoso", "gratuito", o carente de correspondencia con la realidad. La evidencia de que el conocimiento humano está, per se, constreñido por un sujeto no debería haber sido históricamente un obstáculo para captar plenamente otra evidencia: la de que siempre es relativo necesariamente a un objeto independiente del sujeto, es decir, cuyo ser no depende de la mera percepción. De manera similar, el conocimiento tiene necesidad tanto de la razón como de la experiencia, y no puede reducirse a ninguna de ellas. Como hemos visto en el apartado 4.2.1., tan necesaria es la teoría para sostener la observación, como lo es la experiencia para fundamentar la teoría.

Dicho esto, debo añadir que, aunque la definición de verdad como adecuación o correspondencia es nítida, no garantiza, por supuesto, que ese concepto pueda ser aplicado al modo del método empírico para determinar cuando una afirmación es verdadera o no lo es. Si nos descubrimos intentando buscar una especie de método científico universal capaz de proporcionarnos certeza empírica acerca de la verdad de una parte de nuestro conocimiento, entonces esto será señal de que seguimos pensando otra vez a través de las categorías del positivismo y de que continuamos atrapados en su marco conceptual.

Por si acaso fuera necesario, permítaseme una ulterior aclaración. En modo alguno, estoy cuestionando la validez de los métodos empíricos para determinar lo que es verdadero o no respecto en muchos aspectos de la realidad. Es obvio, por ejemplo, el método científico es capaz de proporcionar suficiente certeza, en muchos casos, respecto a cuál de las distintas pretensiones de verdad es verdadera, cuando se trata de algunas realidades físicas. Pero también debería ser obvio que: a) la realidad no puede quedar confinada solo a la experiencia (por ejemplo, ser amable es una cualidad tan real y verdadera como ser alto, aunque, a diferencia de lo segundo, lo primero no puede ser verificado empíricamente $\mathrm{y}$, por consiguiente, no produce la certeza máxima); b) el método científico no es competente en absoluto para establecer la verdad en muchos otros aspectos de la realidad, cuyo ser no puede ser reducido a la mera experiencia (el "mundo de la vida" -por usar la expresión de Husserl- es realmente muy diferente del mundo físico) ${ }^{10}$.

\section{Conclusiones}

A lo largo de estas páginas, han ido surgiendo un buen número de asuntos cruciales, que merecerían mayor consideración. Sin embargo, llega el momento de poner fin a nuestro recorrido intentando sintetizar ahora las conclusiones más relevantes que pueden extraerse de la investigación realizada.

para conocer 'con verdad' es negada” (MuÑOZ-TORRES, 2007: 236-7). Este deseo por el más alto grado de seguridad respecto a la validez de nuestro conocimiento puede también ser muy contraproducente. Dicho con palabras atribuidas a GOETHE: "si no pretendiéramos conocer todo con tanta exactitud, es muy probable que conociéramos mejor las cosas".

10 Basta con pensar que, en nuestra vida diaria, no solemos recurrir a ningún tipo de método científico para determinar si nuestro conocimiento es verdadero o falso (aunque esto no significa, por supuesto, que éste esté siempre libre de error). 
1.Como hemos visto, la objetividad es, frecuentemente, un concepto insuficientemente definido; o, al menos, su significado es dado por supuesto muchas veces, por lo que suele permanecer difuso en muchos debates académicos y profesionales. Esto se debe, en parte, a los diversos cambios que ha sufrido a lo largo de su historia. También, al desconocimiento profundo de sus fundamentos filosóficos, que son simplemente ignorados o preteridos la mayor parte de las veces. Como consecuencia de esta situación, el término "objetividad" abarca normalmente muchos significados diferentes que tienden a entremezclar indebidamente aspectos epistemológicos y éticos.

2. La principal fuente de malentendidos y confusión sobre la teoría de la objetividad procede de la aceptación acrítica de las premisas básicas del positivismo y, particularmente, de su dicotomía hecho-valor, que fue transferida al periodismo a través de la distinción entre textos periodísticos considerados factuales y no valorativos (hard news) frente a los textos interpretativos y de opinión.

3. Como he argumentado antes, el dualismo que separa hechos de valores es constitutivamente erróneo, al menos, por las razones siguientes:

a. establecer que algo es un hecho requiere necesariamente una idea de hecho. En otras palabras, este concepto no es factual, sino pre-factual, lo que significa que presupone un conjunto de ideas previas sin las cuales la misma noción de hecho no puede ni siquiera ser entendida.

b. dado que el mundo nos ofrece infinitos hechos, cuyo conocimiento es inabarcable, se impone necesariamente hacer una selección de entre todos ellos. Esta selección siempre es realizada por alguien, desde un punto de vista, con arreglo a ciertos valores y en vista de algunos fines. Sin valores y sin fines, todos los hechos valdrían lo mismo; en definitiva, se volverían irrelevantes.

c. la asunción básica del positivismo, a saber, que el conocimiento, para ser objetivo, debe estar exento de valores, no es, en absoluto, un juicio fáctico, puesto que no procede de la experiencia, ni puede ser verificado a través de ella. Por tanto, esta premisa encierra implícitamente una contradicción flagrante.

4. Es obvio que cualquier acto de conocimiento es, intrínsicamente y por definición, una relación bipolar entre un sujeto y objeto. Sin ninguno de estos dos términos, no puede haber conocimiento en absoluto. Ahora bien, haciendo un paralelismo entre conocimiento y otros tipos de relación, como la maternidad biológica, preguntarse cómo un sujeto puede conocer objetivamente equivale a inquirir cómo una mujer puede ser madre biológica sin tener hijos. En consecuencia, la teoría de la objetividad parte de una pregunta mal concebida, que desafía en vano las leyes de la lógica.

5. Con la aceptación general de sus premisas básicas, el positivismo ha logrado crear en nuestras mentes un esquema conceptual omnipresente que identifica erróneamente objetividad con verdad y subjetividad con ausencia de ella, estableciendo así un falso dilema entre sujeto y objeto. Igualmente, ha dado por sentados otros falsos dilemas, que desvirtúan la naturaleza del conocimiento humano, como el que enfrenta razón a experiencia intentando reducirlo a una de ambas.

6. De la refutación del pensamiento positivista, no se sigue la validez del relativismo, que pone todo el énfasis en el sujeto, en detrimento del objeto de conocimiento. La corriente relativista, que ha crecido vigorosamente en las últimas décadas en mu- 
chos campos académicos -también en el del estudio sobre los medios- ha fracasado al intentar ofrecer una solución para el problema de la verdad, haciendo que muchos profesionales, abjurando de la objetividad, se hayan pasado a una especie de "anarquismo epistemológico", y sostengan que no es posible ofrecer una representación verdadera sobre el mundo y la vida.

7. En contra de lo que puede parecer a primera vista, positivismo y relativismo forman parte del mismo marco conceptual, que ha convertido en muy inadecuadas las nociones de "objetivo" y "subjetivo" en aras a entender lo que es el conocimiento, dado que este no puede ser "reducido" a ninguno de estos dos términos sin dejar de existir.

8. Una vez que el problema del conocimiento se plantea en términos dualistas de objetividad frente a la subjetividad, se cierran todas las posibilidades de una solución satisfactoria. El único posible camino para salir de la aporía sería, en mi opinión, abandonar el paradigma actual y reemplazarlo por uno nuevo -necesitado con urgencia-, libre tanto de los lazos del positivismo como del relativismo.

Finalmente, a la vista de las deficiencias teóricas del paradigma periodístico de la objetividad, quisiera concluir abogando por la necesidad de que éste quede definitivamente superado y sea sustituido paulatinamente por un nuevo paradigma. Para ello, me aventuro ahora a sugerir dos primeros pasos en la que considero buena dirección: a) reconsiderar y mostrar con frecuencia los errores y falsos dilemas implícitos en el positivismo y en el relativismo; $\mathrm{yb}$ ) repensar las antiguas evidencias sobre las que se funda el realismo clásico de origen aristotélico, que nunca entendió la verdad o el conocimiento en términos de dilema entre objetividad o subjetividad, sino como correspondencia entre mente y realidad, en la que tanto experiencia como razón juegan un papel conjunto definitivo. Será necesario, por supuesto, un gigantesco esfuerzo colectivo de reflexión para alcanzar esta meta, que, probablemente, requerirá también un cambio cultural tan profundo como el que el positivismo consiguió extender al conjunto de la sociedad. Tal vez sea ésta la única manera de que algún día quedemos libres de la extenuante e inútil tarea de empujar la pesada roca de la objetividad, una y otra vez, por toda la eternidad.

\section{Referencias bibliográficas}

ALTHEIDE, David (1976): Creating reality: How TV news distorts events. Beverly Hills, CA, Sage.

BELL, Martin (1998): “The Truth is Our Currency". Harvard International Journal of Press/Politics, 3 (1), 102-09.

CUNNINGHAM, Brent (2003: "Re-thinking Objectivity". Columbia Journalism Review, 42 (2), 24-32.

DONSBACH, Wolfgang \& KLETT, Bettina (1993): "Subjective Objectivity. How Journalists in Four Continents Define a Key Term of their Profession". Gazette, 51, 53-83. 
DURHAM, Meenakshi (1998): "On the relevance of standpoint epistemology to the practice of journalism: The case for «strong objectivity»". Communication Theory, 8, 117-140.

GAUTHIER, Gilles (1993): "In Defence of a Supposedly Outdated Notion: The Range of Application of Journalistic Objectivity". Canadian Journal of Communication, 18, (4), 497-505.

GLASSER, Theodore (1992): “Objectivity and News Bias”, in: Elliot D. Cohen (Ed.), Philosophical Issues in Journalism, New York, Oxford University Press, 176-85.

HACKETT, Robert (1984): "Decline of a Paradigm? Bias and Objectivity in News Media Studies". Critical Studies in Mass Communication, 1, 229-259.

HARDING, Sandra (1991): Whose science? Whose knowledge?: Thinking from women's lives. Ithaca, NY, Cornell University Press.

HEMPEL, Carl (1965): Aspects of Scientific Explanation. New York, The Free Press.

KAPLAN, Richard (2002): Politics and the American Press: The Rise of Objectivity, 1865-1920. New York, Cambridge University Press.

KLAIDMAN, Stephen \& BEAUCHAMP, Tom (1987): The Virtuous Journalist. New York, Oxford University Press.

KOVACH, Bill \& ROSENSTIEL, Tom (2001): The Elements of Journalism. New York, Three Rivers Press.

MACINTYRE, Alasdair (2007 [1981]): After Virtue. Notre Dame, University of Notre Dame Press.

MEYER, Philip (2002 [1991]): Precision Journalism. 4ª ed., Lanham, MD, Rowman and Littlefield. (ed. or.: The New Precision Journalism).

MINDICH, David (1998): Just the Facts: How “Objectivity” Came to Define American Journalism. New York, New York University Press.

MUÑOZ-TORRES, Juan Ramón (1995): “Objetivismo, subjetivismo y realismo como posturas epistemológicas sobre la actividad informativa". Comunicación y Sociedad, VIII (2), 141-171.

MUÑOZ-TORRES, Juan Ramón (2002): “Objetividad y verdad. Sobre el vigor contemporáneo de la falacia objetivista". Revista de Filosofía. Universidad Complutense de Madrid, 27 (I), 161-190.

MUÑOZ-TORRES, Juan Ramón (2007): “Underlying Epistemological Conceptions in Journalism". Journalism Studies, 8 (2), 224-247.

NAGEL, Thomas (1997): The Last Word. New York, Oxford University Press.

PUTNAM, Hilary (1981): Reason, Truth and History. Cambridge, Cambridge University Press.

PUTNAM, Hilary (2002): The collapse of the fact/value dichotomy and other essays. Cambridge, Massachusetts, Harvard University Press. 
REESE, Stephen (1990): “The News Paradigm and the Ideology of Objectivity: A Socialist at The Wall Street Journal". Critical Studies in Mass Communication, 7, 390-409.

RICH, Carole (1997): Writing and Reporting News. CA, Wadsworth.

RYAN, Michael (2001): "Journalistic Ethics, Objectivity, Existential Journalism, Standpoint Epistemology, and Public Journalism". Journal of Mass Media Ethics, $16(1), 3-22$.

ROSHCO, Bernard (1975): Newsmaking. Chicago, University of Chicago Press.

ROSEN, Jay (1993): "Beyond Objectivity. It is a Myth, an Important One, but Often Crippling and It Needs to Be Replaced With a More Inspiring Concept". Nieman Reports, 47 (4), 48-53.

SCHUDSON, Michael (1978): Discovering the News. New York, Basic Books.

WARD, Stephen (1999): "Pragmatic News Objectivity: Objectivity With a Human Face" (paper D-37). The Joan Shorenstein Center Press Politics and Public Policy.

WARD, Stephen (2005): The Invention of Journalism Ethics. The Path To Objectivity And Beyond. Montreal,: McGill-Queen's University Press. 